\title{
Optimal multi-objective distributed generation location and capacity by elite strategy genetic algorithm considering timing characteristics
}

\author{
WEI Xinquan ${ }^{1 *}$, DUAN Xiangjun ${ }^{2}$, CHEN Lei $^{3}$, ZHENG Weiyan $^{4}$ \\ ${ }^{1}$ State Grid Shanghai Energy Interconnection Research Institute Co., Ltd., Shanghai, 201210, China \\ ${ }^{2}$ China Electric Power Research Institute Co., Ltd., Beijing, 100085, China \\ ${ }^{3}$ State Grid Zhejiang Electric Power Co., Ltd., Hangzhou, Zhejiang Province, 310007, China \\ ${ }^{4}$ State Grid Zhejiang Electric Power Co., Ltd. Hangzhou Power Supply Company, Hangzhou, Zhejiang Province, 310016, China
}

\begin{abstract}
In this paper, a distributed generation location and capacity optimization model considering the probability of scenario occurrence is established. The optimization objective is to minimize the total cost of investment, annual power loss of distribution network and node voltage deviation. The improved genetic algorithm with elitist retention mechanism is used to solve the model. The IEEE33 system is used to show the location and constant capacity of the distributed power supply under different conditions. It shows that the reasonable and optimized configuration of the distributed power supply can obtain better voltage quality and minimize the cost function, which verifies the effectiveness of the proposed model.
\end{abstract}

\section{Introduction}

The distributed power supply can reduce the power loss in the transmission lines, support the voltage of each load node and improve the power supply reliability ${ }^{[1]}$. At the same time, the distributed generation has the characteristics of random fluctuation, and a large number of access will aggravate the voltage fluctuation of distribution network ${ }^{[2]}$.

Liu $^{[3]}$ established a DG location and capacity model with operation risk cost as the objective function, with DG access capacity, active power balance and node voltage limit as the constraints. Sun ${ }^{[4]}$ considered voltage stability and current margin, and adopted multi-objective decision-making method to optimize the configuration of DG access. Zhu ${ }^{[5]}$ established the DG optimal allocation model considering the dynamic reconstruction of the network.

Due to the uncertainty of random fluctuation of DG and load, many scholars consider the timing characteristics of DG and load ${ }^{[6][7]}$. Heuristic algorithms such as cat swarm algorithm ${ }^{[8]}$, particle swarm algorithm $^{[9]}$, genetic algorithm ${ }^{[10]}$ are widely used to solve the DG configuration problem.

In this paper, $k$-means clustering algorithm is used to extract the scene division and probability of DG active power and load change mode. A multi-objective optimization model is established to minimize the investment cost, annual power loss and node voltage deviation. The impact of scenario occurrence probability on DG configuration is considered in the objective function. The improved genetic algorithm with elitist mechanism is used to solve the model, and compared with the existing model to verify the effectiveness of the model.

\section{Multi objective location and capacity determination}

\subsection{Objective function}

The objective function $F_{l}$ represents the cost function.

$$
\min F_{1}=C_{n} \cdot \frac{r(1+r)^{n_{0}}}{(1+r)^{n_{0}}-1}
$$

Where $C_{n}$ is the initial investment cost of wind power and photovoltaic, $r=8 \%$ is the discount rate. $n_{0}$ is the service life of wind turbine and photovoltaic. $C_{o}$ is the operation and maintenance cost obtained by scenario division.

The objective function $F_{2}$ is the annual electric energy loss of the distribution network after connecting to DG. Where $W_{i, k}$ represents the power loss in line $i$ under scenario $k$.

$$
\min F_{2}=\sum_{k=1}^{K} p(k) \sum_{i}^{L} W_{i, k}
$$

The objective function $F_{3}$ is the total node voltage deviation $\Delta U$ of the distribution network after connecting

*Corresponding author's e-mail: xinquan1103@126.com 
to DG. $U_{i}$ is the actual value of node voltage, and $U_{e}$ is the rated voltage of the node.

$$
\min F_{3}=\sum_{i=1}^{N}\left|\frac{U_{i}-U_{e}}{U_{e}}\right| \times 100 \%
$$

\subsection{Constraints}

The constraints of optimization model are shown in formula (4)-(6). The power balance constraints for each node are as follows.

$$
\left\{\begin{array}{l}
P_{i}^{k}=U_{i}^{k} \sum_{j \in C_{i}} U_{j}^{k}\left(G_{i j} \cos \theta_{i j}^{k}+B_{i j} \sin \theta_{i j}^{k}\right) \\
Q_{i}^{k}=U_{i}^{k} \sum_{j \in C_{i}} U_{j}^{k}\left(G_{i j} \sin \theta_{i j}^{k}-B_{i j} \cos \theta_{i j}^{k}\right)
\end{array}\right.
$$

Where $U_{i}^{k}$ and $U_{j}^{k}$ is the voltage value of node $i . C_{i}$ is the set of nodes adjacent to node $i . G_{i j}$ and $B_{i j}$ is the real part and the imaginary part of the admittance matrix, and $\theta_{i j}^{k}$ is the voltage phase angle difference between nodes $i$ and $j$. Voltage constraint is as follows.

$$
U_{i}^{\min } \leq U_{i} \leq U_{i}^{\max }, i=1,2, \ldots, N
$$

$U_{i}^{\min }$ and $U_{i}^{\max }$ is the minimum and maximum allowable node voltage. The branch power constraint is as follows.

$$
P_{l} \leq P_{l}^{\max }, l=1,2, \ldots N_{l}
$$

\subsection{Improved genetic algorithm}

In this paper, an improved genetic algorithm with elite strategy was used to solve the problem ${ }^{[11]}$. Genetic algorithm (GA) is a kind of heuristic algorithm based on the theory of biological evolution. Individuals with high fitness were selected through crossover and mutation. Repeated iterations make the population evolve and finally converge to the global optimal solution.

The elitist retention strategy can prevent the optimal individual from being destroyed by crossover and mutation operations. Individuals with the best fitness value in the evolution process are directly copied to the next generation.

\section{Case study}

IEEE33 node system is used as an example to verify the model, as shown in Figure 1. The head end reference voltage of the network is $12.66 \mathrm{kV}$. The system power reference value is 10MVA. The total network load is $5084.26+\mathrm{j} 2547.32 \mathrm{kVA}$, and the branch impedance and node load are shown in literature ${ }^{[6]}$.

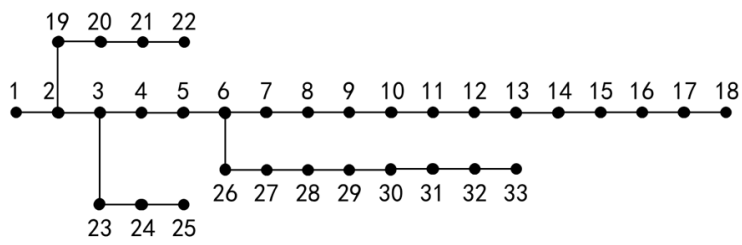

Figure 1 IEEE33 system node diagram
Figure 2 shows the comparison of convergence rates of different algorithms. The elitist retention strategy guarantees the global convergence of genetic algorithm, and the convergence speed is faster than standard genetic algorithm and PSO algorithm.

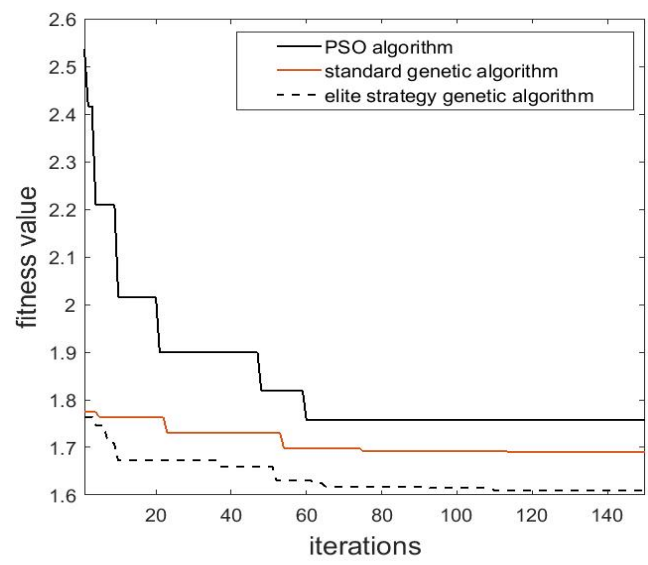

Figure 2 Comparison of convergence rates of different algorithms

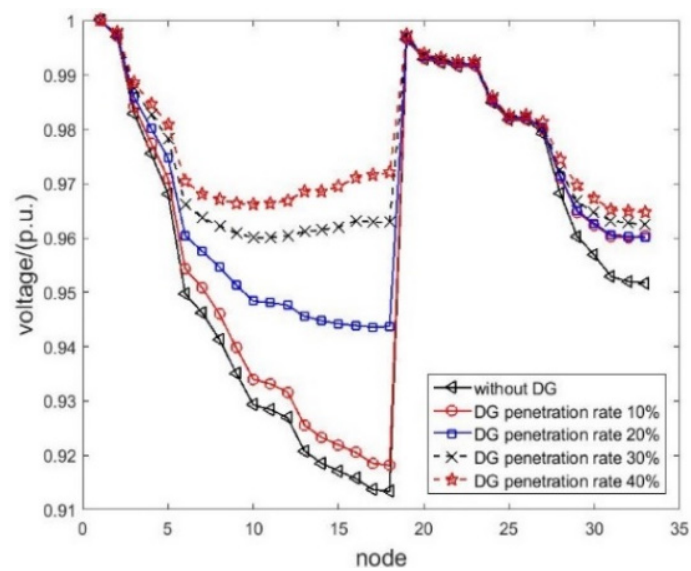

Figure 3 Effect of different permeability on node voltage offset

Figure 3 shows the node voltage offset curve of the optimal DG configuration under different permeability. When the permeability increases, the voltage offset of each node decreases. The larger the permeability is, the smaller the node voltage offset is.

\section{Conclusion}

In this paper, a multi-objective optimization model for distributed generation location and capacity is established. The objective is to minimize the cost, annual power loss and node voltage deviation. The improved genetic algorithm with elitist retention mechanism is used to solve the model. The IEEE33 node system is simulated and the optimized location and capacity of distributed power supply are given. 


\section{Acknowledgement}

This paper was supported by the science and technology project of the headquarters of State Grid Corporation of China (project code: 5400-201919144A-0-0-00).

\section{References}

1. Yang G, Guan L, Lin LX. Analysis of Influences of Grid-connected Distributed Generation on Feeder Power Supply Ability in Distribution Network[J]. Proceedings of the CSU-EPSA, 2020,32(1):13-20.

2. Chen Y, Liu DC, Wu J. Research on influence of distributed photovoltaic generation on voltage fluctuations in distribution network[J]. Electrical Measurement \& Instrumentation, 2018,055(014):2732.

3. Liu XS, Wang LX, Wu YB. Locating and Sizing Planning of Distributed Generation Power Supply Considering the Operational Risk Cost of Distribution Network[J]. Transactions of China Electrotechnical Society, 2019,34(z1):264-271.

4. Sun SX, Li XM, Liu ZS. Research on locating and sizing of DG in the distribution network considering voltage stability assessment[J]. Power System Protection and Control, 2019, 47(03):94-100.

5. Junpeng Z, Wei G U, Handan Z. Optimal Siting and Sizing of Distributed Generators Considering
Dynamic Network Reconfiguration[J]. Automation of Electric Power Systems, 2018, 042(005):111-119.

6. Chu Z, Li Z, Bai WW. Optimal siting and sizing of distributed generations considering uncertainties and environmental factors $[\mathrm{J}]$. Power System Protection and Control, 2017,45(13):34-41.

7. Li L, Tang W, Bai M. Multi-objective locating and sizing of distributed generators based on timesequence characteristics[J]. Automation of Electric Power Systems, 2013, 37(3):58-63+128.

8. Yang L, Yang XH, Wu Y. Research on optimized distributed generations locating based on modified cat swarm optimization[J]. Power System Protection and Control, 2019, 047(1):95-100.

9. Wang XL, Zhao XY, Cao JW. Optimization design for locating and sizing of distributed generation based on improved particle swarm algorithm[J]. Journal of Dalian University of Technology, 2014,(3):355-360.

10. Ma L, Liu JP. Multi-objective planning of multi-type distributed generation considering timing characteristics and environmental benefits[J]. Power System Protection and Control, 2016,44(19):32-40.

11. J. Chen, Z. Xiao. Research on adaptive genetic algorithm based on multi-population elite selection strategy[C]. 2017 2nd IEEE International Conference on Computational Intelligence and Applications (ICCIA), Beijing, 2017, 108-112. 